\title{
Higgs boson searches at LEP II
}

\author{
David Smith \\ Department of Physics and Astronomy \\ University of Glasgow \\ Kelvin Building \\ University Avenue \\ Glasgow \\ G12 $8 Q Q$ \\ $U K$ \\ E-mail: D.H.Smith@cern.chi
}

Abstract: A brief overview of current Higgs boson searches at LEP II is presented, illustrated with some of the details of the analyses used in the search for the Standard Model Higgs boson. Following this, recent preliminary results from LEP are reported. The new data set which is examined is the $\sim 900 p b^{-1}$ of data which have been collected at LEP during the year 1999 by the four collaborations. The LEP working group for Higgs boson searches has combined these data with data sets collected earlier at lower energies. None of the searches found a statistically significant excess above the Standard Model background prediction. Resulting $95 \%$ confidence level bounds are presented here.

\section{Motivation for the Standard Model Higgs boson search}

The Standard Model of Elementary Particle Interactions has been remarkably successful in reproducing and predicting the behavior of the observed fundamental constituents of matter. The only elements of the Standard Model yet to be observed are the $\tau$ neutrino ${ }^{1}$ and the Higgs boson. The Higgs boson is of considerable interest as it arises as a consequence of the Higgs mechanism, the process through which the fundamental particles in the model acquire mass. The mass of the Higgs boson itself is a free parameter of the theory. Once the Higgs boson mass is known all other properties of the Higgs particle such as production cross sections and decay branching ratios are calculable.

Although the Higgs boson mass is not predicted by the Standard Model it may be con-

\footnotetext{
${ }^{1}$ At the time of writing (Autumn 2000) the DONUT collaboration have observed direct evidence for the $\tau$ neutrino at Fermilab. See the collaboration home page on the world wide web at URL http://fn872.fnal.gov/
}

strained by fitting the model to the observed electroweak data. In electroweak fits measured parameters are allowed to vary within their errors and hence indirectly place limits on the range that the mass of the Higgs boson could take to remain compatible with the measurements[i].

Figure 1 i' shows the result of this fit for $m_{h}$, the Higgs boson mass. The most likely mass obtained in this way is $\sim 70 \mathrm{GeV} / c^{2}$. The upper mass limit at $95 \%$ confidence level is $185 \mathrm{GeV} / c^{2}$. It can be seen that the fit only produces a loose determination of $m_{h}$, given that the Higgs boson mass enters the electroweak radiative corrections only as a logarithmic term. If the experimentally excluded range of $m_{h}$ is included into the statistical interpretation then the $95 \%$ confidence level upper limit from the electroweak fit rises to $280 \mathrm{GeV} / c^{2}$.

Although the constraints are not very tight it can be seen that the data clearly favor a light Higgs boson. 


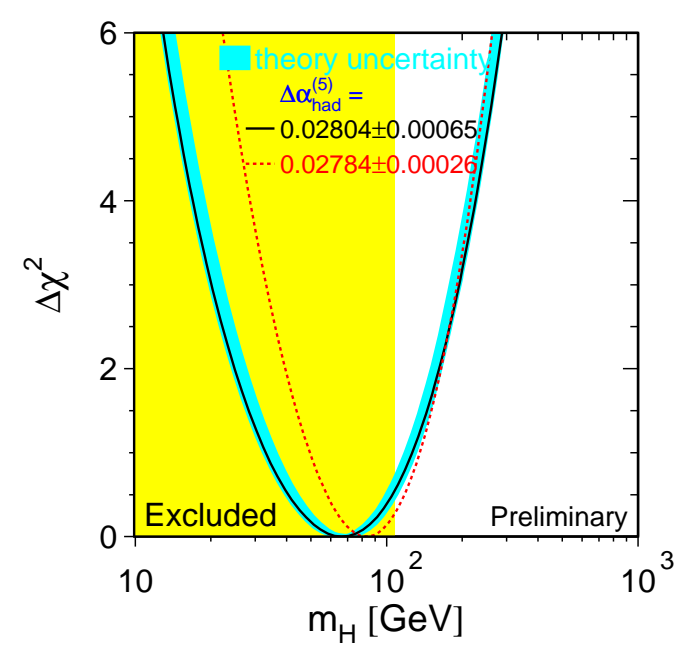

Figure 1: Electroweak fit for the mass of the Standard Model Higgs boson. The shaded region labeled Excluded corresponds to the lower limit set on the Higgs boson mass by direct searches. The fit alone gives an upper mass limit of $185 \mathrm{GeV} / c^{2}$ at $95 \%$ confidence level on the Higgs boson mass.

\section{Standard Model Higgs boson pro- duction at LEP II}

At the LEP collider the period of running since 1996 has been known as LEP II and throughout this period the center of mass energy in the $e^{+} e^{-}$ collisions has been gradually increased. During the recent running period, in $1999,{ }^{2}$ the collider has seen energies in the range $192 \mathrm{GeV}<\sqrt{s}<$ $202 \mathrm{GeV}$. The $\sqrt{s}$ is the highest ever at LEP and represents the culmination of great effort on the part of the accelerator division. The reason behind the continued drive towards the highest collision energies has been the search for new physics and the search for the Standard Model Higgs boson.

Currently for a Standard Model Higgs boson to be produced in any detectable quantity at LEP, $m_{h} \leq 110 \mathrm{GeV} / c^{2}$. Therefore the production diagrams and decay fractions quoted here are typical for $m_{h} \sim 100 \mathrm{GeV} / c^{2}$. The dominant production process is the Higgsstrahlung di-

\footnotetext{
${ }^{2}$ At the time of writing LEP has nearly finished the year 2000 run. Presently $\sqrt{s}$ is around $207 \mathrm{GeV}$. The running period was recently extended by a month to gain more data in order to investigate a possible Standard Model Higgs boson signal $\sim 115 \mathrm{GeV} / c^{2}$.
}

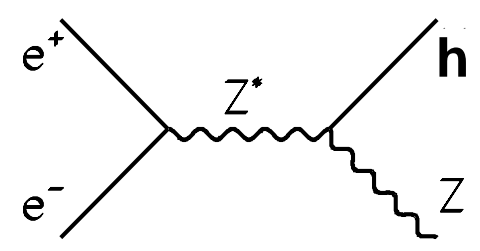

Figure 2: The main Higgs boson production diagram for the $e^{+} e^{-}$LEP collider when $m_{h} \sim$ $100 \mathrm{GeV} / c^{2}$. The digram is often called Higgsstrahlung due to the radiation of the Higgs boson from the $\mathrm{Z}^{*}$.

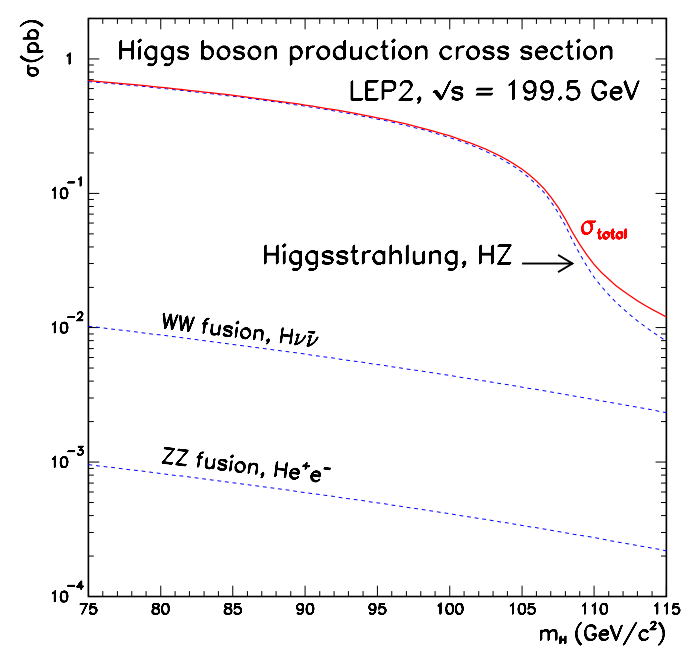

Figure 3: Cross section for the $e^{+} e^{-}$production of the Higgs boson of the Standard Model against Higgs boson mass. The center of mass energy is assumed to be $199.5 \mathrm{GeV}$. Three diagrams are considered: the Higgsstrahlung of figure ble (fusion) diagrams where the Higgs boson may be produced. The cross section for Higgsstrahlung drops rapidly with $m_{h}$ once the kinematic threshold for $\mathrm{hZ}$ production at $m_{h}=\sqrt{s}-m_{Z}$ is crossed.

agram. (see figure $\overline{2}$ ')

There are two additional diagrams, WW and $\mathrm{ZZ}$ fusion, which contribute to the $h \nu_{e} \overline{\nu_{e}}$ and $h e^{+} e^{-}$final states, respectively. However, in the region where the searches are sensitive to Higgs boson detection, the fusion processes contribute only a small fraction to the total production cross section. In figure $\overline{\beta_{1}}$ can be seen the cross section for Standard Model Higgs boson production at one particular center of mass energy. 


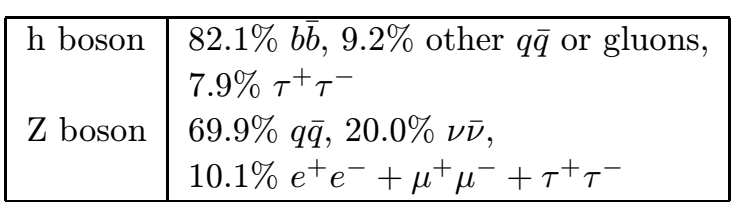

Table 1: Decay branching fractions for the $\mathrm{Z}$ boson and for a Standard Model Higgs boson $m_{h}=$ $100 \mathrm{GeV} / c^{2}$.

\begin{tabular}{|c|c|c|c|}
\hline$h \ell^{+} \ell^{-}$ & $X \tau^{+} \tau^{-}$ & $h \nu \bar{\nu}$ & $(q \bar{q} / g g) q \bar{q}$ \\
\hline \hline $6.7 \%$ & $8.9 \%$ & $20.0 \%$ & $63.8 \%$ \\
\hline
\end{tabular}

Table 2: The branching ratios for the four main hZ final states. The values are for $m_{h}=100 \mathrm{GeV} / c^{2}$. Here $\ell$ denotes an $e$ or $\mu$ charged lepton. $\mathrm{X}$ denotes a $\tau^{+} \tau^{-}$or $q \bar{q}$ pair, covering the cases where either the $\mathrm{Z}$ boson, $\mathrm{h}$ boson or possibly both decay to the charged $\tau$ lepton.

\section{Looking for the Standard Model Higgs boson}

Experimentally the search for the Standard Model Higgs boson falls naturally into four topologically distinct final states, according to the decay of the $\mathrm{h}$ and $\mathrm{Z}$ bosons from the Higgsstrahlung production. Decay branching fractions are given in table $\overline{1} 1$.

Four final final states are identified in table 글, together with their associated branching fraction which follow from the $\mathrm{h}$ and $\mathrm{Z}$ boson decays. Typically each final state has one analysis designed to select the hZ signal over Standard Model background.

Due to the large branching fraction of $h \rightarrow b \bar{b}$ $b$ tagging is used in nearly all analysis. B tagging is the experimental determination that a hadronic jet originated from the hadronisation of a bottom quark and subsequent decay of the $\mathrm{B}$ hadron $(b$ jet $)$. The $\mathrm{b}$ tag is the single most important tool to reduce the search backgrounds. There are several methods used to identify b jets, but the most powerful relies on the non-zero (albeit small) lifetime of the B hadron, $\sim 1.5 \mathrm{ps}$. At LEP II energies this corresponds to a typical flight length of several $\mathrm{mm}$ for the hadron. With very accurate charged particle tracking this decay length may be directly reconstructed, and b jets can be separated from lighter quark (udsc) and gluon jets.

\section{Results of the search for the Stan- dard Model Higgs boson}

During 1999 the LEP experiments ALEPH, DELPHI, L3 and OPAL collected $\sim 900 p b^{-1}$ between them. The data has been combined among the experiments and also with data recorded earlier at lower center of mass energies. The combination was performed by The LEP working group for Higgs boson searches, and all the results presented here are taken from the working group

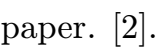

Due to the very rapid decrease in production cross section for Higgsstrahlung when approaching kinematic threshold the Standard Model Higgs boson search is limited by energy more than luminosity. Hence significant improvement in sensitivity was possible with the addition of the new data due to the increase in $\sqrt{s}$ over that of previous data sets. The additional luminosity gained by incorporating the previous data sets is significant, however, when considering the Minimal Supersymmetric Standard Model.

The mass plot of some of the selected Standard Model Higgs boson candidates from the 1999 data is shown in figure

While it is interesting to look at the mass plot of observed candidates a statistically rigorous procedure is required. For this reason the mass plot is illustrative only, and a formalism is developed to express the results of the search in terms of classical confidence levels.

A test statistic, $Q$, is used to rate the experimentally observed data set in terms of how 'signal like' the data are. The test statistic is a function of the number of observed events, the number of expected signal and background events and of other discriminating variables. Discriminating variables include the reconstructed Higgs boson mass and $b$ tag values for an event. In this way the test statistic uses more information than is

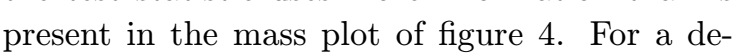
tailed discussion and definition of $Q$ see $[2 \overline{2}]$.

In order to test for indication of a Higgs boson signal in the data the value of $\mathrm{CL}_{\mathrm{b}}$ (defined in figure $\overline{5_{1}}$ text), is found for each Higgs mass hypothesis. A deviation of $\mathrm{CL}_{\mathrm{b}}$ from the expected value of $50 \%$ indicates a excess or deficit with respect to the expected background prediction. 


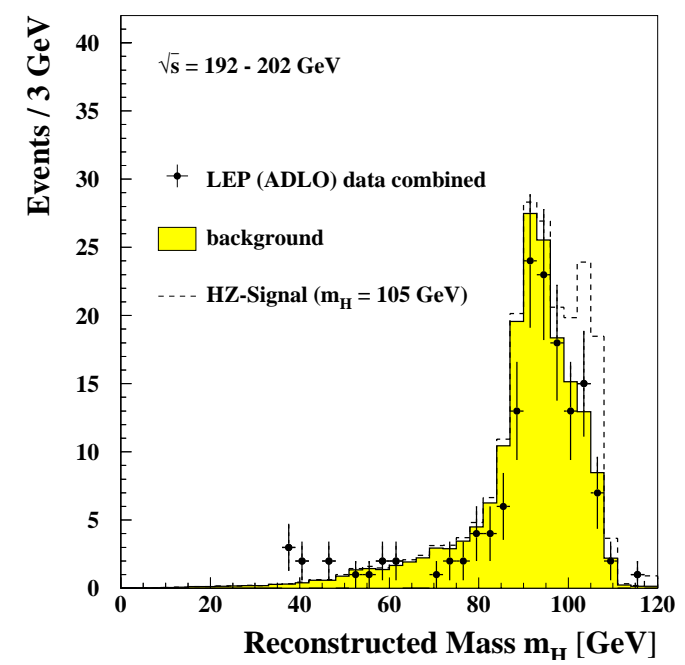

Figure 4: The set of points with error bars show the distribution of reconstructed Higgs mass for some of the selected Higgs boson candidate events from the LEP 1999 data set. The solid histogram shows the corresponding distribution of the Standard Model background prediction. The dashed histogram shows the expected distribution of a Higgs boson signal, $m_{h}=105 \mathrm{GeV} / c^{2}$ added on the top of the background prediction.

\begin{tabular}{|c|c|c|}
\hline & Observed & Expected \\
\hline Mass Limit $\mathrm{GeV} / c^{2}$ & 107.9 & 109.1 \\
\hline
\end{tabular}

Table 3: The lower mass limits set on the Higgs boson of the Standard Model at 95\% confidence level.

In figure $\overline{6}_{1}^{\prime}$ the value of $1-\mathrm{CL}_{\mathrm{b}}$ is plotted against the hypothesised Higgs boson mass, $m_{H}$. It can be seen that at no point is there a significant deviation away from the background prediction by more than about $1 \sigma$. For this reason it is concluded that no significant production of Higgs bosons has been observed.

In the absence of Higgs boson production a lower limit on the Higgs boson mass is set. The quantity $\mathrm{CL}_{\mathrm{s}}$ is computed, where $\mathrm{CL}_{\mathrm{s}}=$ $\mathrm{CL}_{\mathrm{s}+\mathrm{b}} / \mathrm{CL}_{\mathrm{b}}$. The lower limit on the Higgs boson mass at $95 \%$ confidence level is the value of the mass hypothesis for which $\mathrm{CL}_{\mathrm{s}}$ exceeds $5 \%$. The lower mass limits set on the Higgs boson from the LEP data are shown in table

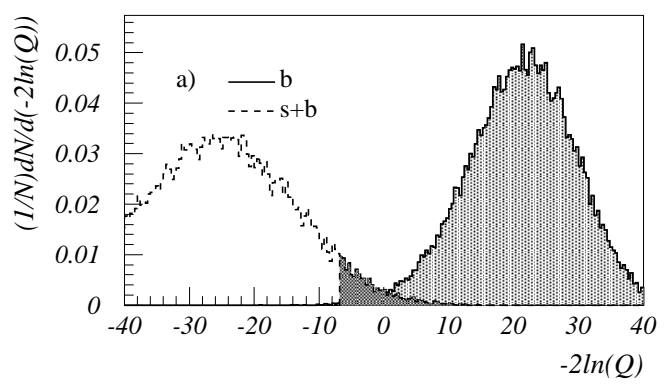

Figure 5: A plot of the distribution of test statistics for many simulated data sets. For a signal like data set the plotted quantity tends to a negative value, and for a background like set it tends to the positive. The two distinct distributions correspond to two large collections of simulated data sets, the hatched with background only, the clear with background and signal. A separation between the two in terms of the test statistic is seen. The quantities $\mathrm{CL}_{\mathrm{b}}$ and $\mathrm{CL}_{\mathrm{s}+\mathrm{b}}$ may then be defined for a given data set. $\mathrm{CL}_{\mathrm{b}}\left(\mathrm{CL}_{\mathrm{s}+\mathrm{b}}\right)$ is the fraction of the simulated data sets containing background only (background and signal) with a more background like test statistic than the given set.

\section{The neutral Higgs bosons of the MSSM and search results}

The Minimal Supersymmetric Standard Model (MSSM) is an example of a two-doublet Higgs model. In any two-doublet model there are four Higgs bosons. Three neutral: $\mathrm{h}$ and $\mathrm{H}$ (CP-even) and $\mathrm{A}$ (CP-odd), and the charged $\mathrm{H}^{+}, \mathrm{H}^{-}$. The $\mathrm{h}$ is defined to be the lighter of the two CP-even Higgs bosons, so that $m_{h}<m_{H}$.

In the case of the MSSM, $\tan \beta$ is defined to be the ratio of the vacuum expectation value of the two Higgs field doublets, while $\alpha$ is a mixing angle in the CP-even Higgs sector. $m_{h}$ is related to $m_{A}$ by $\tan \beta$.

LEP could be sensitive to $\mathrm{h}$ and A bosons production and a search in terms of $m_{h}$ and $\tan \beta$ has been performed. Production of the $\mathrm{h}$ boson remains through the Higgsstrahlung diagram, the cross section is modified from that of the Standard Model by a factor $\sin ^{2}(\beta-\alpha)$. In addition there is the possibility to pair produce $\mathrm{h}$ and $\mathrm{A}$ bosons. The cross section for hA pair production varies with a factor of $\cos ^{2}(\beta-\alpha)$ and is thus complentary to Higgsstrahlung production. 


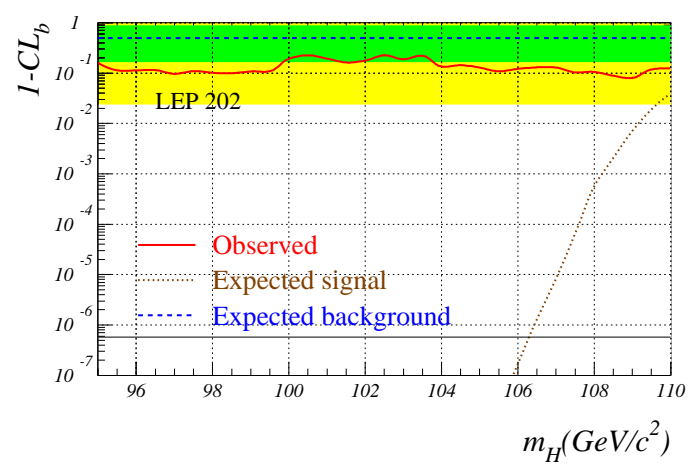

Figure 6: The plot of $1-\mathrm{CL}_{\mathrm{b}}$ against the Higgs boson mass hypothesis, $m_{H}$. The expected value of $\mathrm{CL}_{\mathrm{b}}$ is $50 \%$ and is shown by the dashed line. The solid red line is the observed value of $1-\mathrm{CL}_{\mathrm{b}}$. If the observed value approaches zero (one) it shows that the observed data set show an excess (deficit) with respect to the Standard Model background prediction. The greed and yellow bands indicate the significance of a deviation of $\mathrm{CL}_{\mathrm{b}}$ away from the expected value of 1 and $2 \sigma$ respectively. The horizontal black line at $5.7 \times 10^{-7}$ indicates the level for a $5 \sigma$ discovery. The dotted line is the expected value of $\mathrm{CL}_{\mathrm{b}}$ for data sets in which a Higgs boson signal is present.

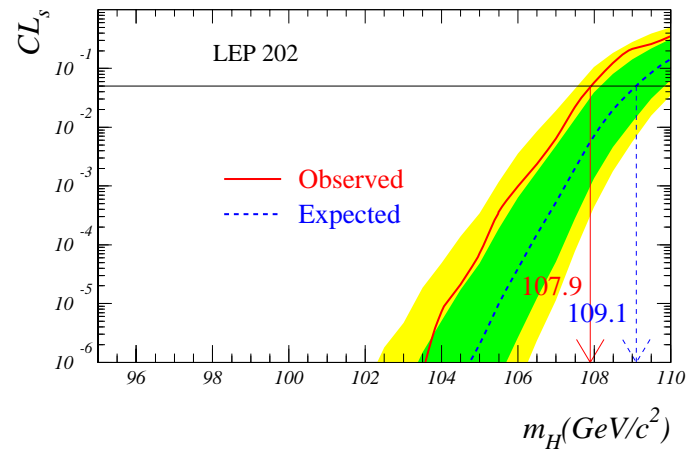

Figure 7: The plot of $\mathrm{CL}_{\mathrm{s}}$ against the Higgs boson mass hypothesis, $m_{H}$. The dashed line shows the expected value of $\mathrm{CL}_{\mathrm{s}}$ from data sets with background only present. The solid red line shows the observed value. The green and yellow bands indicate the significance of a deviation of $\mathrm{CL}_{\mathrm{s}}$ away from the expected value of 1 and $2 \sigma$ respectively. The horizontal black line is drawn at the $5 \%$ level, where the $95 \%$ confidence level is measured.

Both the $\mathrm{h}$ and the A Higgs bosons decay to $b \bar{b}$ or $\tau^{+} \tau^{-}$predominantly for a large range of $\tan \beta$ values. Searches are therefore designed to select $b \bar{b} b \bar{b}$ or $b \bar{b} \tau^{+} \tau^{-}$final states.

When the combined LEP data was examined

\begin{tabular}{|l|c|c|}
\hline & Observed & Expected \\
\hline$m_{h}$ & 88.3 & 90.8 \\
$m_{A}$ & 88.4 & 91.1 \\
$\tan \beta$ (no mixing) & $0.4-4.1$ & $0.4-4.6$ \\
$\tan \beta$ (large mixing) & $0.7-1.8$ & $0.6-1.9$ \\
\hline
\end{tabular}

Table 4: The lower mass, $\mathrm{GeV} / c^{2}$, limits set on the neutral Higgs bosons of the MSSM at $95 \%$ confidence level, together with ranges of $\tan \beta$ excluded at the same confidence level. No mixing indicates the assumption that there is no mixing in the scalartop sector, while large mixing indicates a mixing such that the range of $\tan \beta$ excluded is minimised. The $\tan \beta$ limits are valid for top quark masses $\leq 174.3 \mathrm{GeV} / c^{2}$.

it was found that no significant excess was observed and so limits were set on $m_{h}, m_{A}$ and $\tan \beta$. The results of the search for neutral Higgs bosons in the context of the MSSM are summarised in table

\section{Conclusion}

With the increased center of mass energies at the LEP collider 1999 has been a very exciting year for Higgs boson searches. However analysis of the data have failed to reveal evidence of Higgs boson production. In the year 2000 LEP will complete is research programme before the dismantling of the machine and experiments in preparation for the construction of the LHC. The final data collected will be at the highest LEP energies ever, with consequently continuing exciting prospects for Higgs boson searches in the near future. After the end of LEP the Tevatron and eventually the LHC itself with continue the search.

\section{References}

[1] ALEPH, DELPHI, L3 and OPAL collab., The LEP Electroweak Working Group, and the SLD Heavy Flavour and Electroweak Groups, $A$ Combination of Preliminary Electroweak Measurements and Constraints on the Standard Model, CERN-EP/2000-016

[2] ALEPH, DELPHI, L3 and OPAL collab., The LEP working group for Higgs boson searches, Searches for Higgs bosons: Preliminary combined results using LEP data collected at energies up to $202 \mathrm{GeV}$, CERN-EP/2000-055. 